\title{
When cooperators cheat
}

\section{A study of a cuckoo species that usually shows cooperative nesting behaviour, but sometimes cheats at parenthood by} laying eggs in others' nests, reveals the benefits that have shaped the evolution of this parasitic tactic. SEE LETTER P.96

\section{ANDREW G. ZINK \& JOHN M. EADIE}

$\mathrm{T}$ The evolutionary conditions that drive cheating versus cooperative nesting tactics in birds are a major focus of interest in animal-behaviour research. On page 96, Riehl and Strong ${ }^{1}$ report a study of a cuckoo species called the greater ani (Crotophaga major), which sometimes displays a cheating behaviour when nesting called conspecific brood parasitism ${ }^{2,3}$, in addition to cooperative nesting behaviour. The authors tracked the identity of females and the eggs in their nests to assess the costs and benefits of this alternative parasitic tactic.

Conspecific brood parasitism occurs when a female lays her eggs in a nest belonging to another member of the same species, but does not provide any offspring care ${ }^{2,3}$. By contrast, in one specific form of cooperative breeding behaviour, the care and defence of the offspring in a nest are shared between two or more females (and, in some species, their mates $)^{4}$. Crotophaga major is a rare example of a species that exhibits both types of behaviour in the same population, providing an opportunity to examine the evolutionary relationships between these two tactics.

The authors tracked nests in the wild over 11 breeding seasons, and used DNA analysis to identify the birds in each nest and determine the mother of each egg, using techniques that included non-destructive extraction of DNA from eggshell surfaces. Riehl and Strong observed that females almost always show cooperative breeding behaviour at the start of the breeding season. If predators destroyed a nest, the authors found that some affected females pursued a parasitic strategy in the same breeding season, whereas others waited until the next year's breeding season to lay more eggs and nest cooperatively (Fig. 1). The authors report that either cooperative breeding combined with parasitism after nest failure or solely cooperative breeding provided similar numbers of surviving offspring. The parasitic females laid more eggs than the solely cooperative females, but the death rate of parasitic eggs was higher than that of non-parasitic eggs, owing to host rejection. The authors found that any given individual used just one of these two alternative breeding tactics repeatedly over many cases of nest loss.

Three explanations are usually given for

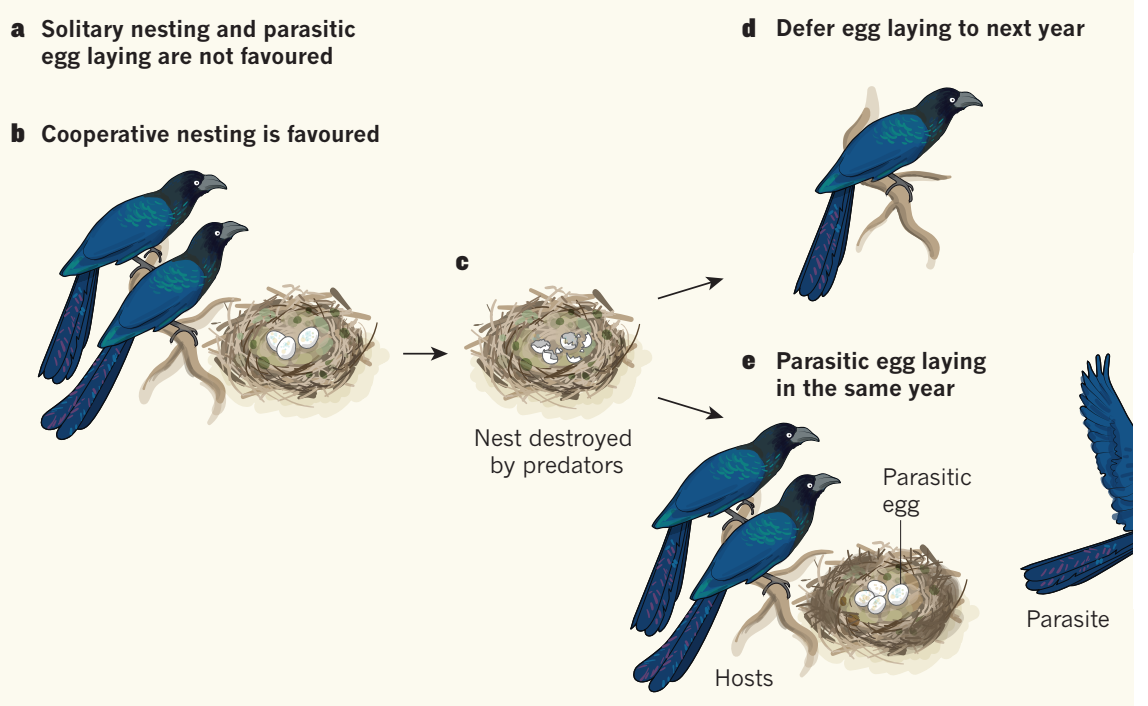

Figure 1 | Nesting strategies of a cuckoo species. Riehl and Strong ${ }^{1}$ report a study of the greater ani (Crotophaga major) in which they tracked the identity of females and the eggs in their nests. a, b, At the start of the annual breeding season, a female bird has three nesting options. a, The two unfavoured options (that less than $2 \%$ of the birds chose) are solitary nesting, in which a single female and her mate care for the nest, and brood parasitism, in which a female lays eggs in a nest and departs without providing offspring care. $\mathbf{b}$, The favoured option is cooperative nesting, in which a nest is shared by two or more females and their mates. c, When cooperative nests were destroyed by predators, some birds deferred egg laying until they bred cooperatively in the next season (d), whereas others changed tactics to parasitic egg laying in the same breeding season (e). Birds following options $\mathbf{d}$ or $\mathbf{e}$ had similar numbers of surviving offspring, and one of the two distinct behaviours was repeatedly chosen by the same individual. The equal fitness of these two behaviours suggests that they have evolved to be maintained as alternative tactics.

why conspecific brood parasitism occurs ${ }^{5}$. One possibility is the 'super mother' scenario in which females develop eggs in excess of the optimal number for their own nest, and lay the extra eggs in other nests. This is a successful strategy in some birds and insects ${ }^{6-8}$. Another explanation, which has had little support ${ }^{9}$, is that the females are specialized parasites that never construct their own nest. The third is that females are making the best of a bad situation in which parasitism is a last resort taken by a female that would otherwise have nested in the usual way ${ }^{10}$. Riehl and Strong provide support for this hypothesis.

Why C. major individuals are not normally parasitic breeders except as a response to nest predation, or why they do not pursue both parasitism and cooperative nesting in the absence of nest predation, is a mystery. Perhaps the benefits of cooperative breeding for egg survival are so great (relative to the lower survival of parasitic eggs associated with host rejection) that it has evolved to be the default option; this would explain why parasitism is pursued only after nest failure, rather than as the sole option of choice or pursued concurrently with cooperative nesting.

More than 300 bird species cooperatively breed, and 200 show conspecific brood parasitism $^{5,9}$, but few species are found to exhibit both. It has been proposed that cooperative breeding and parasitism might represent extremes of offspring care by a female that contributes eggs to a nest already occupied by another female ${ }^{2}$. Cooperative breeding might have evolved directly from parasitism if a host provides incentives to entice a female parasite to remain at the nest and cooperate ${ }^{3}$. However, Riehl and Strong show that the relationship between cooperative breeding and parasitism is 
more complicated than was previously thought, because parasitism seems to have evolved as part of an existing system of cooperative breeding, rather than the other way around. Intriguingly, the same single factor of high levels of nest predation drives both behaviours. Cooperative breeding is favoured over solitary nesting (in which a single female and her mate care for the nest) because of predator pressure ${ }^{4}$.

The idea that genetic relatedness between individuals can affect the evolution of social interactions has had a central role in our understanding of cooperative breeding in many species, and some models ${ }^{2,3}$ suggest that kinship might also have a role in the evolution of brood parasitism. A brood parasite might actively target kin to increase the survival of host eggs by buffering their predation risk as a result of adding parasite eggs ${ }^{11}$, or hosts might accept eggs of non-nesting kin because that is the parasite's only opportunity to reproduce ${ }^{12}$. Parasitism might, therefore, sometimes have a cooperative aspect, blurring the distinction between cooperative breeding and parasitism when kin are involved. However, Riehl and Strong show that kinship does not play a part in the parasitism of $C$. major, because the relatedness of the hosts and parasites was not greater than that in the general population. This meant that the authors could focus on the evolution of nesting tactics without having to consider the influence of kinship.

Why specific C. major females pursue parasitism is unknown. The observation that individual females consistently used this tactic each time their nest failed, whereas others did not, suggests that there might be a heritable basis. Alternatively, parasitism might be shaped by other factors, such as development, learning or physiology. Perhaps certain females consistently provide less parental care than others in cooperatively breeding nests, and therefore have more resources in reserve for parasitic egg laying if their nest is destroyed. Another possibility is that some females avoid parasitism and reserve resources to meet the higher demand for parental care in their own future nests. Quantifying the costs of parental care and the energetic demands of egg laying would help to shed light on this. Following these behaviours across the entire lifetimes of $C$. major could determine whether the benefits of parasitism across breeding seasons found in this study scale up to benefits in lifetime reproductive success in this fascinating species.

Andrew G. Zink is in the Department of Biology, San Francisco State University, San Francisco, California 94132, USA. John M. Eadie is in the Department of Wildlife, Fish \& Conservation Biology, University of California, Davis, Davis, California 95616, USA.

e-mails:zink@sfsu.edu; jmeadie@ucdavis.edu

1. Riehl, C. \& Strong, M. J. Nature 567, 96-99 (2019).

2. Zink, A. G. Am. Nat. 155, 395-405 (2000).

3. Zink, A. G. \& Lyon, B. E. Am. Nat. 187, 35-47 (2016).
4. Riehl, C. Proc. R. Soc. B 278, 1728-1735 (2011)

5. Lyon, B. E. \& Eadie, J. M. Annu. Rev. Ecol. Syst. 39, 343-363 (2008)

6. Åhlund, M. \& Andersson, M. Nature 414, 600-601 (2001).

7. Lyon, B. E. Anim. Behav. 46, 911-928 (1993).

8. Zink, A. G. Behav. Ecol. Sociobiol. 54, 406-415 (2003).
9. Lyon, B. E. \& Eadie, J. M. in Avian Brood Parasitism (ed. Soler, M.) 105-123 (Springer, 2017).

10. Petrie, M. \& Moller, A. P. Trends Ecol. Evol. 6, 315-320 (1991).

11. Loeb, M. L. G. Am. Nat. 161, 129-142 (2003).

12. Andersson, M. Am. Nat. 189, 138-152 (2017).

This article was published online on 27 February 2019.

\section{VIROLOGY}

\section{Receptor bats for the next flu pandemic}

\section{How bat influenza viruses infect cells has been unclear. The discovery that they bind to a cell receptor that is present in many different species raises concerns about their potential risk to humans. SEE LETTER P.109}

\section{SEWENDY S. BARCLAY}

B ats are excellent hosts for viruses: they are numerous, accounting for $20 \%$ of all mammals on Earth, and prolific, existing in colonies of up to 20 million individuals. Bats harbour dangerous pathogens that can spread to domestic animals and humans. Ebola, SARS and Nipah viruses have all crossed from bats to humans, either directly or through intermediate hosts ${ }^{1}$. The discovery in 2012 (ref. 2) that bats harbour influenza A viruses was alarming, because flu viruses are notoriously adept at crossing from animals into humans and causing pandemics that have devastating consequences ${ }^{3}$. Karakus et al. ${ }^{4}$ show on page 109 that bat flu viruses infect animals using a host cell receptor that is highly similar across species. The findings are a key step towards quantifying the risk to human and animal health that is posed by flu viruses residing in bats.
Wild birds are the natural reservoir of most influenza A viruses. Avian flu viruses infect birds by binding to sialic acid receptors on the host cells (Fig. 1). The cells that line the human respiratory tract also display sialic acid receptors, but these are slightly different from the receptors in birds. Avian flu viruses can acquire the capacity to pass through the air between humans when they undergo mutations in haemagglutinin, a glycoprotein, which forms the spikes on the virus particle that interact with the sialic acid receptors on host cells. The requirement for optimal receptor binding is a major barrier to infection between species that saves us from frequent flu pandemics originating from birds ${ }^{3}$.

Until the discovery of bat flu viruses, all known influenza A viruses used sialic acid receptors to infect their hosts. It was a huge surprise when studies revealed that bat flu viruses did not use sialic acid receptors to

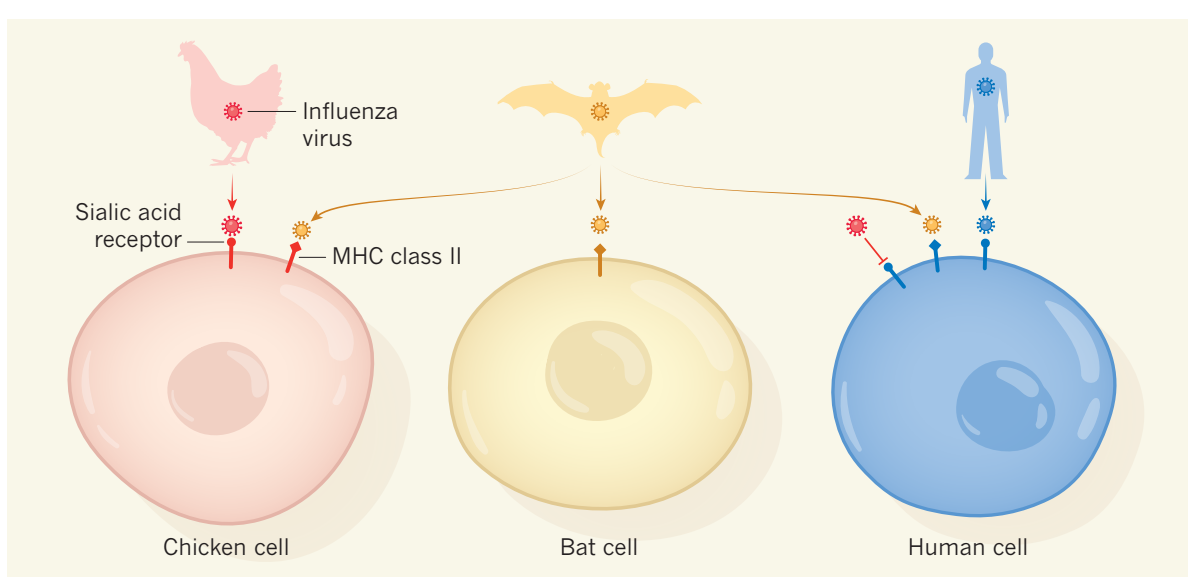

Figure 1 | Infection by influenza viruses. Karakus et al. ${ }^{4}$ report that the bat flu virus can use a protein complex known as major histocompatibility complex (MHC) class II from different species as a receptor by which to enter cells and infect the host. This contrasts with avian and human flu viruses, which bind to sialic acid receptors on cells. The avian virus does not efficiently use the human sialic acid receptor, and so does not easily infect human cells. 